\title{
SOME RESULTS FOR QUADRATIC ELEMENTS OF A BANACH ALGEBRA
}

\author{
M. T. KARAEV and S. PEHLIVAN \\ Department of Mathematics, Süleyman Demirel University, Cunur Campus, 32260 Isparta, Turkey \\ e-mail: garayev@fef.sdu.edu.tr,serpil@sdu.edu.tr
}

(Received 19 March, 2003; accepted 5 May, 2004)

\begin{abstract}
Several properties of some quadratic elements of a unitial Banach algebra are studied. Deddens subspaces are also introduced and discussed.
\end{abstract}

2000 Mathematics Subject Classification. Primary 46H10. Secondary 47A15.

1. Introduction. Let $\mathcal{A}$ be a complex Banach algebra with unit $e$. An element $a \in \mathcal{A}$ is called quadratic if it satisfies $a^{2}+\lambda_{1} a+\lambda_{2} e=0$ for some scalars $\lambda_{1}$ and $\lambda_{2}$. Observe that idempotents and nilpotent elements of order 2 are quadratic elements.

Our main goal in this paper is to study some properties of such elements. See $[\mathbf{1}, \mathbf{2}, \mathbf{3}, \mathbf{4}]$ for concrete applications.

2. Deddens subspaces. Let $\mathcal{A}$ be a Banach algebra with a unit $e$. For any two invertible elements $a_{1}, a_{2} \in \mathcal{A}$ put

$$
\mathcal{D}_{a_{1}, a_{2}} \stackrel{\text { def }}{=}\left\{x \in \mathcal{A}: \sup _{n \geq 0}\left\|a_{1}^{n} x a_{2}^{-n}\right\| \stackrel{\text { def }}{=} c_{x}<\infty\right\} .
$$

We call the subspaces $\mathcal{D}_{a_{1}, a_{2}}$ and $\mathcal{D}_{a_{2}, a_{1}}$ the Deddens subspaces. Note that, when $a_{1}=$ $a_{2}$ the notion of Deddens subspace coincides with the notion of Deddens algebra, introduced in [4].

Our main result in this section is the following theorem.

THEOREM 1. Let $\mathcal{A}$ be a Banach algebra with unit e. Let $p$ be any idempotent and $q$ a nilpotent of order 2 , respectively. We have

(a) $\mathcal{D}_{e+p, e+q}=\{x \in \mathcal{A}: p x=x q\}$,

(b) $\mathcal{D}_{e+q, e+p}=\{x \in \mathcal{A}: q x=x p\}$.

Proof. (a) Let us denote Intertw $\{p, q\}=\{x \in \mathcal{A}: p x=x q\}$. The inclusion Intertw $\{p, q\} \subset \mathcal{D}_{e+p, e+q}$ is obvious. To prove the reverse inclusion $\mathcal{D}_{e+p, e+q} \subset$ Intertw $\{p, q\}$, let $x \in \mathcal{D}_{e+p, e+q}$ be any element. Putting

$$
c_{n}=a_{1}^{n} x a_{2}^{-n} \quad(n \geq 0),
$$

where $a_{1}=e+p, a_{2}=e+q$, we deduce that

$$
\left\|c_{n}\right\| \leq c_{x} \quad(n \geq 0)
$$


We have

$$
c_{n} a_{2}=a_{1}^{n} x a_{2}^{-n} a_{2}=a_{1}\left(a_{1}^{n-1} x a_{2}^{-n+1}\right)=a_{1} c_{n-1} ;
$$

that is

$$
c_{n} a_{2}=a_{1} c_{n-1} \quad(n \geq 1) .
$$

From (2) we obtain

$$
c_{n} a_{2}^{n}=a_{1}^{n} c_{0} \quad(n \geq 1)
$$

or

$$
c_{n}(e+q)^{n}=(e+p)^{n} c_{0}=\left(e+\left(2^{n}-1\right) p\right) x \quad(n \geq 1)
$$

that is

$$
c_{n}=\left(e+\left(2^{n}-1\right) p\right) x(e-n q) \quad(n \geq 1) .
$$

From this equality, we deduce that

$$
c_{n}-x=\left(2^{n}-1\right) p x-n x q-n\left(2^{n}-1\right) p x q,
$$

for all $n \geq 1$. By taking the equality (1) into account, it follows from (3) that

$$
\|p x q\| \leq \frac{\left\|c_{n}-x\right\|}{n\left(2^{n}-1\right)}+\frac{\|p x\|}{n}+\frac{\|x q\|}{2^{n}-1} \rightarrow 0
$$

as $n \rightarrow \infty$. Hence $p x q=0$, and therefore

$$
c_{n}-x=\left(2^{n}-1\right) p x-n x q .
$$

From this we obtain

$$
\|p x\| \leq \frac{\left\|c_{n}-x\right\|}{2^{n}-1}+\frac{n}{2^{n}-1}\|x q\| \rightarrow 0
$$

as $n \rightarrow \infty$. Hence $p x=0$. Therefore $c_{n}-x=-n x q$, which implies that

$$
\|x q\|=\frac{\left\|c_{n}-x\right\|}{n} \rightarrow 0 \quad \text { as } n \rightarrow \infty
$$

and so $x q=0$. Hence $c_{n}-x=0 \quad(n \geq 1)$. In particular $c_{1}=x$, so that $x=$ $(e+p) x(e-q)$. Hence, $(e+p) x=x(e+q)$. Therefore $p x=x q$, which means that $x \in \operatorname{Intertw}\{p, q\}$, and so $\mathcal{D}_{e+p, e+q} \subset \operatorname{Intertw}\{p, q\}$, which completes the proof of (a).

(b) The proof is very similar to that of (a) and is omitted.

Corollary 2. Let $\mathcal{A}$ be a complex Banach algebra with unit e. Let $p$ be any idempotent and $q$ a nilpotent of order 2 , respectively. We have

$$
\left(\mathcal{D}_{e+p, e+q} \cap \mathcal{D}_{e+q, e+p}\right) \cap\{p\}^{\prime}=\left(\mathcal{D}_{e+p, e+q} \cap \mathcal{D}_{e+q, e+p}\right) \cap\{q\}^{\prime} .
$$

Here $\{t\}^{\prime}$ stands for the commutant of $t$. 
Let $\mathcal{A}$ be a Banach algebra with the idempotent $p$ and with a unit $e$. Define the set $S_{p}$ as follows:

$$
S_{p}=\{x \in \mathcal{A}: p x(e-p)=0\} .
$$

By analogy with the proof of Theorem 1, we can state directly the following theorem.

THEOREM 3. Let $\mathcal{A}$ be a Banach algebra with an idempotent $p$ and with a unit e. Then $\mathcal{D}_{e+p, e+p}$ is an algebra and $\mathcal{D}_{e+p, e+p}=S_{p}$; thus the Deddens algebra $\mathcal{D}_{e+p, e+p}$ coincides with the algebra $S_{p}$.

Proof. It follows from the definition of Deddens subspaces that $\mathcal{D}_{e+p, e+p}$ is an algebra. For the second statement of the theorem, it is easy to check that $(e+p)^{-1}=$ $e-\frac{1}{2} p$. Therefore

$$
(e+p)^{n}=e+\left(2^{n}-1\right) p \quad(n \geq 0)
$$

and

$$
(e+p)^{-n}=e+\left(\frac{1}{2^{n}}-1\right) p \quad(n \geq 0) .
$$

By setting

$$
c_{n}=(e+p)^{n} x(e+p)^{-n} \quad(n \geq 0),
$$

where $x$ is any element of $\mathcal{A}$, we obtain

$$
c_{n}(e+p)=(e+p)\left[(e+p)^{n-1} x\left(e-\frac{1}{2} p\right)^{n-1}\right]=(e+p) c_{n-1}
$$

for every $n \geq 1$, which implies that

$$
c_{n}(e+p)^{n}=(e+p)^{n} c_{0} \quad(n \geq 0) .
$$

By applying equalities (4), (5) we have

$$
c_{n}=x+\left(\frac{1}{2^{n}}-1\right) x p+\left(2^{n}-1\right) p x+\left(2^{n}-1\right)\left(\frac{1}{2^{n}}-1\right) p x p,
$$

$n=0,1,2, \ldots$ Then, for every $x \in \mathcal{D}_{e+p, e+p}$, it follows that

$$
\begin{aligned}
0 & =\lim _{n \rightarrow \infty} \frac{1}{\left(2^{n}-1\right)\left(\frac{1}{2^{n}}-1\right)}\left(c_{n}-x\right) \\
& =\lim _{n \rightarrow \infty}\left(\frac{1}{\frac{1}{2^{n}}-1} p x+\frac{1}{2^{n}-1} x p+p x p\right) \\
& =p x p-p x
\end{aligned}
$$


or equivalently

$$
p x(e-p)=0
$$

i.e., $x \in S_{p}$.

Conversely, if $x \in S_{p}$, then again it is clear from the equality (6) that

$$
\begin{aligned}
\left\|c_{n}\right\| & =\left\|x+\left(\frac{1}{2^{n}}-1\right) x p+\frac{2^{n}-1}{2^{n}} p x p\right\| \\
& \leq\|x\|+\|x p\|+\|p x p\| \\
& =c_{x}<+\infty
\end{aligned}
$$

for any $n \geq 0$. Hence $x \in \mathcal{D}_{e+p, e+p}$.

Prior to stating two corollaries of Theorem 3, we require some terminology and notation.

Let $\mathcal{A}$ be a complex Banach algebra with unit $e$. An element $a \in \mathcal{A}$, is said to be a regular von Neumann element if there exists $b \in \mathcal{A}$, such that $a=a b a$.

It is obvious that the invertible elements of $\mathcal{A}$ are regular von Neumann elements. Also, in the special case in which $\mathcal{A}=\mathcal{B}(H)$, the algebra of bounded linear operators on a complex Hilbert space $H$, all isometries of $\mathcal{B}(H)$ are regular von Neumann elements of $\mathcal{B}(H)$.

For any non invertible regular von Neumann element $a$, define

$$
q_{a}=a(e-a b), \quad p_{a}=a b,
$$

where $b \in \mathcal{A}$ and $a=a b a$. Clearly $q_{a}^{2}=0$ and $p_{a}^{2}=p_{a}$.

COROllary 4. Let $\mathcal{A}$ be a complex Banach algebra with unit e. Then $\mathcal{D}_{e+p_{a}, e+p_{a}}=S_{p_{a}}$.

Proof. This follows at once from Theorem 3.

Finally, we give one more result on quadratic elements $q_{a}$ and $p_{a}^{\perp} \stackrel{\text { def }}{=} e-p_{a}$.

Proposition 5. Let $\mathcal{A}$ be a Banach algebra with unit e. Suppose that $x, y, a \in \mathcal{A}$ are elements such that a is a regular element and

$$
x a-a y=q_{a} x .
$$

If $\sigma(x)=\{0\}$ (that is, $x$ is a quasinilpotent element), then $\sigma\left(p_{a}^{\perp} x\right)=\{0\}$.

Proof. By induction on $n$, we prove that

$$
\left(p_{a}^{\perp} x\right)^{n}=p_{a}^{\perp} x^{n},
$$

for every $n \geq 1$. For $n=1$, the assertion is obvious. Let $n>1$ and let $\left(p_{a}^{\perp} x\right)^{n-1}=$ $p_{a}^{\perp} x^{n-1}$. The regularity of element $a$ implies that $p_{a}^{\perp} a=0$. Then by using the condition (7) we have

$$
\begin{aligned}
\left(p_{a}^{\perp} x\right)^{n} & =p_{a}^{\perp} x\left(p_{a}^{\perp} x\right)^{n-1}=p_{a}^{\perp} x p_{a}^{\perp} x^{n-1}=p_{a}^{\perp} x(e-a b) x^{n-1} \\
& =p_{a}^{\perp} x^{n}-p_{a}^{\perp} x a b x^{n-1}=p_{a}^{\perp} x^{n}-p_{a}^{\perp}\left(a y+q_{a} x\right) b x^{n-1} \\
& =p_{a}^{\perp} x^{n}-p_{a}^{\perp} a\left(y+p_{a}^{\perp} x\right) b x^{n-1}=p_{a}^{\perp} x^{n} .
\end{aligned}
$$

Thus, the equality (8) is proved. From (8) the assertion of the proposition is obvious. This completes the proof. 
REMARKS $(a)$. We recall that for any two elements $x, a$ of the Banach algebra $\mathcal{A}$, the well-known "Kleinecke-Shirokov" condition $[x,[x, a]]=0$ implies the quasinilpotency of the commutator $[x, a] \stackrel{\text { def }}{=} x a-a x$. See [5], [6]. In particular, the condition

$$
[x, a]=x
$$

implies that $[x, a]$ is a nilpotent element. It is known that (9) is not a necessary condition for the nilpotency of $[x, a]$. The condition (7) of Proposition 5, in particular, gives such an example. Indeed, the relation(7) implies that $(x a-a y)^{2}=0$. Therefore, when $y=x$, $(x a-a x)^{2}=0$, but clearly $x a-a x=q_{a} x \neq x$.

(b). It should be mentioned that the statement of the type " $\sigma(x)=\{0\} \Rightarrow \sigma(a x)=$ $\{0\}$ " is of importance in many problems of Banach algebra theory and operator theory. The Shulman's paper [6] is a good reference in this sense. In particular, in [6] the following question is raised.

QUESTION. Let elements $x, a$ of Banach algebra satisfy the conditions

$$
[x,[x, a]]=0
$$

and $\sigma(x)=\{0\}$. Is it true that $\sigma(a x)=\{0\}$ ?

3. Reducing subspaces. Let $H$ be a complex Hilbert space and $\mathcal{B}(H)$ the algebra of bounded linear operators on $H$.

Corollary 6. AlgLat $\mathcal{Q}=\bigcap_{E \in \text { Lat } \mathcal{Q}} \mathcal{D}_{I+P_{E}, I+P_{E}}$, where $\mathcal{Q}$ is a subset of $\mathcal{B}(H)$, Lat $\mathcal{Q}$ the lattice of closed subspaces $E$ of $H$ invariant under $\mathcal{Q}, P_{E}$ is the orthogonal projection of $H$ onto $E$ and $A \lg L a t \mathcal{Q}=\{T \in \mathcal{B}(H): T E \subseteq E$ for all $E \in L a t \mathcal{Q}\}$.

Proof. This follows at once from Theorem 3.

We recall that a reducing subspace of a bounded linear operator $T$ on $H$ is a common invariant subspace for $T$ and $T^{*}$. It is known that a subspace $E \subset H$ is a reducing subspace for $T$ if and only if $T P_{E}=P_{E} T$, where $P_{E}$ is the orthogonal projection of $H$ onto $E$.

Allan and Zemanek proved in [2, Corollary 9] that every quadratic operator on $H$ has a reducing subspace. Our next theorem describes the reducing subspaces of a nilpotent operator on a Hilbert space $H$ in terms of $C_{Q}$ classes. We first recall the definition of the $C_{Q}$ class. Let $S$ be a positive linear operator on a Hilbert space $H$. There are positive real numbers $m$ and $M$ and $Q$ in $\mathcal{B}(H)$ such that $0<m I \leq S \leq M I$ and $Q=S^{-1 / 2}$. Then

$$
C_{Q}=\left\{T \in \mathcal{B}(H): Q T^{n} Q=P_{H} U^{n} \mid H, n=1,2, \ldots\right\},
$$

where $U$ is a unitary operator on some Hilbert space $K \supset H$. Note that $T \in C_{Q}$ if and only if $T$ satisfies the condition:

$$
(S h, h)+2 \operatorname{Re}(z(I-S) T h, h)+|z|^{2}((S-2 I) T h, T h) \geq 0,
$$

for any $h \in H$ and $z \in \mathbb{C},|z| \leq 1$. The classes $C_{Q}$ were defined by Langer. See [7, p. 55].

THEOREM 7. Let $N \in \mathcal{B}(H)$ be a nilpotent operator. The subspace $E \subset H$ is a reducing subspace of the operator $N$ if and only if $E=T^{k-1} H$, for some operator 
$T$ belonging to some class $C_{Q}$ and for some integer $k \geq 2$ satisfying $T^{k}=(I+N) T$ $(I+N)^{-1}$.

Proof. The first part of the theorem is obvious. Indeed, if $E \subset H$ is a reducing subspace of $N$, then $P_{E} N=N P_{E}$, where $P_{E}$ is the orthogonal projection of $H$ onto $E$, and hence, $E=P_{E} H, T=P_{E}, Q=I$ and $k=2$.

We now prove the "only part" of the theorem.

From the condition $T^{k}=(I+N) T(I+N)^{-1}$ it is easy to see that

$$
T^{k^{n}}(I+N)=(I+N) T^{k^{n-1}}
$$

for all $n \geq 1$ Since $T \in C_{Q}$, for some $Q$, then we have that

$$
\left\|T^{k^{n}}\right\| \leq\left\|Q^{-1}\right\|^{2}
$$

and hence, from (10) and (11) by the result of Deddens and Wong [8, Lemma 2] we assert that $T^{k}=T$, see also [4, Lemma 2]. Therefore $T N=N T$, and hence, $T^{k-1} N=$ $N T^{k-1}$. On the other hand,

$$
T^{2(k-1)}=T^{k} T^{k-2}=T T^{k-2}=T^{k-1} ;
$$

that is, $T^{k-1}$ is a projection. $T^{k-1}$ is an orthogonal projection, by [9], since $T \in C_{Q}$ and so the equality $T^{k-1} N=N T^{k-1}$ means that the subspace $T^{k-1} H$ reduces $N$; that is, $E$ reduces $N$ which completes the proof of theorem.

Before passing to the next result, we recall the following definition.

Definition ([10]). The operator $T \in \mathcal{B}(H)$ is called quasidiagonal if there exists a non-decreasing sequence $\left\{P_{n}\right\}_{n \geq 1}$ of finite-dimensional orthogonal projections, for which $P_{n} \rightarrow I$ (strongly) and $\left\|T P_{n}-P_{n} T\right\| \rightarrow 0$ as $n \rightarrow \infty$.

Herrero [11] defined the notion of module of quasidiagonality:

$$
q d(T)=\liminf _{\substack{P \\ P \rightarrow I}}\|T P-P T\|,
$$

where $\mathcal{P}$ is an ordered (with respect to natural order) set of all finite-dimensional orthogonal projections in $H$. It is known [11] that $T$ is a quasidiagonal operator if and only if $q d(T)=0$. The following theorem was proved by Arora and Sahdev in [12].

TheOrem 8. Let $T \in \mathcal{B}(H), \operatorname{ker} T^{*} \neq\{0\}$ and $C=\inf _{\|x\|=1}\|T x\|>0$. Then $q d(T) \geq C$.

According to a result of Allan and Zemanek [2, Example 6] there is an operator $R$ on $H$, with $R^{2}=0$, but having no finite-dimensional reducing subspace. In the remainder of this section, as an illustration of Theorem 8 , we give an example (see Example 10 below) of a family $\left\{T_{\alpha}\right\}$ of operators on a Hilbert space $H$, with no finitedimensional reducing subspace, converging to the nilpotent operator (with the index of nilpotency2) with finite-dimensional reducing subspace. However, we first prove the following proposition.

Proposition 9. Let $V, W \in \mathcal{B}(H)$ be operators such that $V$ is a nonunitary isometry, $V W=W V\left(\right.$ i.e., $\left.W \in\{V\}^{\prime}\right)$ and $\|W\|<1$. Let us consider the operator $N_{V, W} \stackrel{\text { def }}{=} V\left(I-W V V^{*}\right)$. Then $q d\left(N_{V, W}\right) \geq 1-\|W\|$. 
Proof. Since $\|W\|<1$, the operators $I-W V V^{*}$ and $I-V V^{*} W^{*}$ are invertible. Then we have

$$
\operatorname{ker} N_{V, W}^{*}=\operatorname{ker}\left(I-V V^{*} W^{*}\right) V^{*}=\operatorname{ker} V^{*}=(V H)^{\perp} \neq\{0\}
$$

and

$$
\begin{aligned}
C & =\inf _{\|x\|=1}\left\|N_{V, W} x\right\| \\
& =\inf _{\|x\|=1}\left\|V\left(I-W V V^{*}\right) x\right\| \\
& =\inf _{\|x\|=1}\left\|\left(I-W V V^{*}\right) x\right\| \\
& \geq \inf _{\|x\|=1} \frac{\|x\|}{\left\|\left(I-W V V^{*}\right)^{-1}\right\|} \\
& =\frac{1}{\left\|\left(I-W V V^{*}\right)^{-1}\right\|}>0 .
\end{aligned}
$$

Hence, the conditions of Theorem 8 are valid for the operator $N_{V, W}$. Then, by applying Theorem 8, we have

$$
\begin{aligned}
q d\left(N_{V, W}\right) & \geq \frac{1}{\left\|\left(I-W V V^{*}\right)^{-1}\right\|} \\
& =\frac{1}{\left\|\sum_{n \geq 0}\left(W V V^{*}\right)^{n}\right\|} \\
& =\frac{1}{\| I+\left(\sum_{n \geq 1}\left(W^{n} V V^{*}\right) \|\right.} \\
& =\frac{1}{\left\|I+\left(\sum_{n \geq 1} W^{n}\right) V V^{*}\right\|} \\
& \geq \frac{1}{\sum_{n \geq 0}\|W\|^{n}} \\
& =1-\|W\|,
\end{aligned}
$$

which completes the proof.

EXAMPLE 10. Let $N_{V, \alpha}=V\left(I-\alpha V V^{*}\right)$, where $\alpha$ is a scalar, $|\alpha|<1$ and $V \in \mathcal{B}(H)$ is an isometry.

(i) Each of the operators $N_{V, \alpha} \quad(|\alpha|<1)$ does not have any finite-dimensional reducing subspace.

(ii) $N_{V, \alpha}$ converges to $N_{V}=V\left(I-V V^{*}\right)$ in the uniform operator topology as $\alpha \rightarrow 1^{-}$.

(iii) $N_{V}$ has a finite-dimensional reducing subspace.

Proof. (i) Indeed, by Proposition $9 q d\left(N_{V, \alpha}\right) \geq 1-|\alpha|$, and hence, by the definition of the value $q d\left(N_{V, \alpha}\right)$, each of the operators $N_{V, \alpha}$, where $|\alpha|<1$, has no finite-dimensional reducing subspace.

(ii) From the equality

$$
N_{V, \alpha},=\alpha N_{V}+(1-\alpha) V
$$


it follows that $N_{V, \alpha} \rightarrow N_{V}$ as $\alpha \rightarrow 1^{-}$in the uniform operator topology. Evidently, $N^{2}{ }_{V}=0$.

(iii) For arbitrary fixed $0 \neq x \in \operatorname{ker} V^{*}$ let $E_{x}=\operatorname{span}\{x, V x\}$. Then it is easy to verify that $N_{V} E_{x} \subset E_{x}$ and $N_{V}^{*} E_{x} \subset E_{x}$. Indeed, $N_{V} x=V\left(I-V V^{*}\right) x=V x \in$ $E_{x}, N_{V} V x=V\left(I-V V^{*}\right) V x=0 \in E_{x}$, and hence, $N_{V} \operatorname{span}\{x, V x\} \subset \operatorname{span}\{x, V x\}$, i.e., $N_{V} E_{x} \subset E_{x}$. On the other hand, $N_{V}^{*} x=\left(I-V V^{*}\right) V^{*} x=0 \in E_{x}, N_{V}^{*} V x=$ $\left(I-V V^{*}\right) V^{*} V x=\left(I-V V^{*}\right) x=x \in E_{x}$, and so, $N_{V}^{*} E_{x} \subset E_{x}$. Hence $E_{x}$ reduces $N_{V}$ and $\operatorname{dim} E_{x}=2$, which completes the proof.

4. Other properties. In this section we collect some other properties of the operators $N_{V, W}\left(W \in\{V\}^{\prime}\right)$.

Proposition 11. Let $\alpha$ be a scalar and $V, W \in \mathcal{B}(H)$ operators such that $V$ is an isometry and $W \in\{V\}^{\prime}$. Then the following statements are true.

(a) $r\left(N_{V, W}\right) \leq r(I-W)$, where $r(T)$ stands for the spectral radius of the operator $T$.

(b) If $\|W\|<1$, then $\operatorname{ker} N_{V, W}^{n}=\operatorname{ker} N_{V, W}$ and $\operatorname{ker} N_{V, W}^{* n}=\operatorname{ker} V^{*}$, for any integer $n \geq 1$.

(c) $r\left(N_{V, \alpha}\right)=|1-\alpha|$.

(d) $\left\|N_{V, \alpha}\right\| \geq|1-\alpha|$.

(e) If $|\alpha|^{2}-2 \operatorname{Re} \alpha \geq 0$, then $\left\|N_{V, \alpha}\right\|=\left(|\alpha|^{2}-2 \operatorname{Re} \alpha+1\right)^{\frac{1}{2}}$.

( $f$ ) $\omega\left(N_{V, \alpha}\right) \leq \frac{|\alpha|}{2}+|1-\alpha|$, where $\omega(T)$ stands for the numerical radius of the operator $T$.

(g) If $0<\alpha<1$, then $1-\alpha \leq \omega\left(N_{V, \alpha}\right) \leq 1-\frac{\alpha}{2}$.

(h) If $\alpha>2$ is any real number, then $r\left(N_{V, \alpha}\right)=\omega\left(N_{V, \alpha}\right)=\left\|N_{V, \alpha}\right\|=\alpha-1$. have

Proof. (a) Since $N_{V, W}=W N_{V}+(I-W) V, W V-V W=0$ and $N_{V} V=0$, we

$$
\begin{aligned}
N_{V, W}^{2} & =\left[W N_{V}+(I-W) V\right]^{2} \\
& =(I-W) W V N_{V}+(I-W)^{2} V^{2} \\
& =(I-W) V\left[W N_{V}+(I-W) V\right] \\
& =(I-W) V N_{V, W},
\end{aligned}
$$

so that $N_{V, W}^{2}=(I-W) V N_{V, W}$. After these simple calculations we conclude that

$$
N_{V, W}^{n}=(I-W)^{n-1} V^{n-1} N_{V, W},
$$

for each $n \geq 1$. Since $V$ is an isometry, by the last equality, it follows that $r\left(N_{V, W}\right) \leq$ $r(I-W)$.

(b) From (12), it is clear that

$$
N_{V, W}^{* n}=\left(I-V V^{*} W^{*}\right)\left(I-W^{*}\right)^{n-1} V^{* n},
$$

for each $n \geq 1$. The condition $\|W\|<1$ ensures invertibility of the operators $(I-W)^{n-1}$ and $\left(I-V V^{*} W^{*}\right)\left(I-W^{*}\right)^{n-1}$, so that the equalities (12),(13) apply.

(c) If $W=\alpha I$, then (12) directly implies that $r\left(N_{V, \alpha}\right)=|1-\alpha|$.

(c) $\Rightarrow$ (d). 
(e) In fact, for every $x \in H,\|x\|=1$, we have

$$
\begin{aligned}
\left\|N_{V, \alpha} x\right\|^{2} & =\left\|V\left(I-\alpha V V^{*}\right) x\right\|^{2} \\
& \left.=\|\left(I-\alpha V V^{*}\right) x\right) \|^{2} \\
& =\left(\left(I-\alpha V V^{*}\right) x,\left(I-\alpha V V^{*}\right) x\right) \\
& =\left(\left(I-\alpha V V^{*}-\bar{\alpha} V V^{*}+|\alpha|^{2} V V^{*} x\right), x\right) \\
& =\left(\left(I-(2 \operatorname{Re} \alpha) V V^{*}+|\alpha|^{2} V V^{*}\right) x, x\right) \\
& =1+\left(|\alpha|^{2}-2 \operatorname{Re} \alpha\right)\left(V V^{*} x, x\right) .
\end{aligned}
$$

Since $|\alpha|^{2}-2 \operatorname{Re} \alpha \geq 0$, we obtain $\left\|N_{V, \alpha}\right\|=\left(|\alpha|^{2}-2 \operatorname{Re} \alpha+1\right)^{\frac{1}{2}}$ by taking the suprema of both sides over all unit vectors $x$ in $H$.

(f) It is known [3] that the numerical range of the nilpotent operator $N_{V}$ is a closed circular disc with center 0 and radius $\frac{1}{2}$. (Note that in the work of Tso and $\mathrm{Wu}$ [13] a more general theorem was proved, describing the numerical range of any quadratic operator on a complex Hilbert space.) Then, taking into account the equality $N_{V, \alpha}=\alpha N_{V}+(1-\alpha) V$, we get the required inequality.

(c), (f) $\Rightarrow$ (g).

(h) Since $r\left(N_{V, \alpha}\right) \leq \omega\left(N_{V, \alpha}\right) \leq\left\|N_{V, \alpha}\right\|$, from (c) and (e) follows (h). The proof of Proposition 11 is completed.

Now we apply the operator $N_{V}$ to estimate the angle between subspaces of a Hilbert space $H$. The angle between subspaces $E_{1} \subset H$ and $E_{2} \subset H$ is determined as follows:

$$
\left\langle E_{1}, E_{2}\right\rangle \in\left[0, \frac{\pi}{2}\right], \quad \cos \left\langle E_{1}, E_{2}\right\rangle=\sup \left\{\frac{|(x, y)|}{\|x\|\|y\|}: x \in E_{1}, y \in E_{2}\right\} .
$$

From the definition it immediately follows that

$$
\begin{aligned}
& \cos \left\langle E_{1}, E_{2}\right\rangle=\sup \left\{\frac{\left\|P_{E_{2}} x\right\|}{\|x\|}: x \in E_{1}\right\}=\left\|P_{E_{2}} P_{E_{1}}\right\|, \\
& \sin \left\langle E_{1}, E_{2}\right\rangle=\inf \left\{\frac{\left\|\left(I-P_{E_{2}}\right) x\right\|}{\|x\|}: x \in E_{1}\right\}=\left\|\mathcal{P}_{E_{1} \| E_{2}}\right\|^{-1},
\end{aligned}
$$

where $P_{E_{i}}(i=1,2)$ are orthogonal projections of $H$ onto $E_{i}(i=1,2)$ and $\mathcal{P}_{E_{1} \| E_{2}}$ is the projection onto $E_{1}$, parallel to $E_{2}$.

Proposition 12. Let $K$ be an arbitrary subspace of a Hilbert space $H$ and let $V_{1}, V_{2} \in \mathcal{B}(H)$ be isometries. Then

$$
\left|\cos \left\langle R\left(V_{1}\right)^{\perp}, K\right\rangle-\cos \left\langle R\left(V_{2}\right)^{\perp}, K\right\rangle\right| \leq\left\|N_{V_{1}}-N_{V_{2}}\right\| .
$$

Proof. We use the arguments of the reference [14]. (See also [15].) Indeed,

$$
\begin{aligned}
\cos \left\langle R\left(V_{2}\right)^{\perp}, K\right\rangle & =\sup _{x \in K} \frac{\left\|P_{\left(V_{2} H\right)^{\perp}} x\right\|}{\|x\|} \\
& =\sup _{x \in K} \frac{\left\|V_{2} P_{\left(V_{2} H\right)^{\perp}} x\right\|}{\|x\|}
\end{aligned}
$$




$$
\begin{aligned}
& =\sup _{x \in K} \frac{\left\|V_{2}\left(I-V_{2} V_{2}^{*}\right) x\right\|}{\|x\|} \\
& =\sup _{x \in K} \frac{\left\|N_{V_{2}} x\right\|}{\|x\|} \\
& \leq \sup _{x \in K} \frac{\left\|\left(N_{V_{2}}-N_{V_{1}}\right) x\right\|+\left\|N_{V_{1}} x\right\|}{\|x\|} \\
& \leq\left\|N_{V_{1}}-N_{V_{2}}\right\|+\sup _{x \in K} \frac{\left\|N_{V_{1}} x\right\|}{\|x\|} \\
& =\left\|N_{V_{1}}-N_{V_{2}}\right\|+\sup _{x \in K} \frac{\left\|V_{1} P\left(V_{1} H\right)^{\perp} x\right\|}{\|x\|} \\
& =\left\|N_{V_{1}}-N_{V_{2}}\right\|+\sup _{x \in K} \frac{\left\|P_{\left(V_{1} H\right)^{\perp}} x\right\|}{\|x\|} \\
& =\left\|N_{V_{1}}-N_{V_{2}}\right\|+\cos \left\langle\left(V_{1} H\right)^{\perp}, K\right\rangle \\
& =\left\|N_{V_{1}}-N_{V_{2}}\right\|+\cos \left\langle R\left(V_{1}\right)^{\perp}, K\right\rangle .
\end{aligned}
$$

Similarly, it can be shown that

$$
\cos \left\langle R\left(V_{1}\right)^{\perp}, K\right\rangle \leq\left\|N_{V_{1}}-N_{V_{2}}\right\|+\cos \left\langle R\left(V_{2}\right)^{\perp}, K\right\rangle .
$$

From these inequalities, we get (14), which completes the proof.

We say that an isometry $V \in \mathcal{B}(H)$ has a finite defect if $\operatorname{dim}(V H)^{\perp}<+\infty$. Let us denote by IFD the set of isometries, with finite defects. Put $\mathcal{N}_{I F D}=\left\{N_{V}: V \in I F D\right\}$, which is a subset of the set of all finite-dimensional operators in $H$. valid:

COROLlary 13. Let $V \in \mathcal{B}(H)$ be an isometry. Then the following inequalities are

$$
\begin{aligned}
\inf _{U \in I F D \cap\{V\}^{\prime}} \cos \left\langle R(U)^{\perp}, R(V)\right\rangle & \leq \operatorname{dist}\left(N_{V}, \mathcal{N}_{I F D \cap\{V\}^{\prime}}\right) \\
& \leq 4 \operatorname{dist}\left(V, I F D \cap\{V\}^{\prime}\right) .
\end{aligned}
$$

Proof. The simple calculations show that

$$
\left\|N_{V_{1}}-N_{V_{2}}\right\| \leq 4\left\|V_{1}-V_{2}\right\|,
$$

for every pair of commuting isometries $V_{1}$ and $V_{2}$. In fact,

$$
\begin{aligned}
\left\|N_{V_{1}}-N_{V_{2}}\right\| & =\left\|V_{1}\left(I-V_{1} V_{1}^{*}\right)-V_{2}\left(I-V_{2} V_{2}^{*}\right)\right\| \\
& =\left\|V_{1}-V_{2}+V_{2}^{2} V_{2}^{*}-V_{1}^{2} V_{1}^{*}\right\| \\
& \leq\left\|V_{1}-V_{2}\right\|+\left\|V_{2}^{2} V_{2}^{*}-V_{1}^{2} V_{2}^{*}+V_{1}^{2} V_{2}^{*}-V_{1}^{2} V_{1}^{*}\right\| \\
& \leq\left\|V_{1}-V_{2}\right\|+\left\|\left(V_{2}^{2}-V_{1}^{2}\right) V_{2}^{*}\right\|+\left\|V_{1}^{2}\left(V_{2}^{*}-V_{1}^{*}\right)\right\| \\
& \leq\left\|V_{1}-V_{2}\right\|+\left\|\left(V_{2}-V_{1}\right)\left(V_{2}+V_{1}\right)\right\|+\left\|V_{2}^{*}-V_{1}^{*}\right\| \\
& \leq\left\|V_{1}-V_{2}\right\|+2\left\|V_{1}-V_{2}\right\|+\left\|V_{1}-V_{2}\right\| \\
& =4\left\|V_{1}-V_{2}\right\| .
\end{aligned}
$$


By inequality (14) we have

$$
\left|\cos \left\langle R(U)^{\perp}, K\right\rangle-\cos \left\langle R(V)^{\perp}, K\right\rangle\right| \leq\left\|N_{V}-N_{U}\right\|,
$$

for any $U \in I F D \cap\{V\}^{\prime}$ and $K \subset H$. Then, by choosing $K=V H$ and using (16), from the last inequality we get (15). This completes the proof.

\section{REFERENCES}

1. E. A. Nordgren, M. Radjabalipour, H. Radjavi and P. Rosenthal, Quadratic operators and invariant subspaces, Studia Math. 88 (1988), 263-268.

2. G. R. Allan and J. Zemanek, Invariant subspaces for pairs of projections, J. London Math. Soc. (2) 57 (1998), 449-468.

3. M. T. Karaev, On numerical characteristics of some operators associated with isometries, Spectral theory of operators and its applications 11 (1997), 90-98 (Russian).

4. M. T. Karaev and H. S. Mustafayev, On some properties of Deddens algebras, Rocky Mountain J. Math. 33 (2003), 915-926.

5. P. R. Halmos, A Hilbert space problem book, Second Edition (Springer-Verlag, 1982).

6. V. S. Shulman, Invariant subspaces and spectral mapping theorems, in Functional Analysis and Operator Theory (Warsaw, 1992) Banach Center Publications No 30 (Polish Acad. Sci., 1994), 313-325.

7. B. Sz-Nagy and C. Foias, Harmonic analysis of operators on a Hilbert space (North Holland, Amsterdam, 1970).

8. J. A. Deddens and T. K. Wong, The commutant of analytic Toeplitz operators, Trans. Amer. Math. Soc. 184 (1973), 261-273.

9. M. T. Karaev and H. S. Mustafaev, On some properties of $C_{\rho}$-class operators, Izvest. AN Azerb. SSR, 1-3, (1995), 45-48 (Russian).

10. P. R. Halmos, Ten problems in Hilbert space, Bull. Amer. Math. Soc. 76 (1970), 887-933.

11. D. A. Herrero, Quasitriangularity, in Approxmation of Hilbert space operators, I. (Pitman, 1982), 135-167.

12. S. C. Arora and K. Sahdev, On quasidiagonal operators-II, J. Indian Math. Soc. 59 (1993), 159-166.

13. S.-H. Tso and P. Y. Wu, Matrical ranges of quadratic operators, Rocky Mountain J. Math. 29 (1999), 1139-1152.

14. V. I. Vasyunin, Unconditional converging spectral expansions and interpolation problems, Trudy MIAN AN SSSR 130 (1977), 5-49 (Russian). 1986).

15. N. K. Nikolski, Treatise on the shift operator, Spectral function theory (Springer-Verlag, 